\title{
Erythraeid mites (Acariformes: Actinedida: Erythraeidae) of the Greater Caucasus region of Azerbaijan
}

\author{
Gulnar A. Alizade \\ Faculty of Biology, Azerbaijan State Pedogogical University, Azerbaijan \\ E-mail: gulnar.alizade1993@mail.ru
}

Received: 03.02.2020 Accepted 03.03.2020

\begin{abstract}
The summary is given in the article about the observed species of the Erythraeidae Robineau- Desvoidy, 1828 mites in the Greater Caucasus Region of Azerbaijan. 25 species belonging to 8 genera of 5 sub-families have been found. Among them 1 sub-family (Balaustiinae Grandjean, 1947), 4 genera (Erythraeus Latreille, 1806, Curteria Southcott, 1961, Balaustium v.Heyden, 1826, Neobalaustium Willmann, 1951) and 22 species are new for the Caucasian fauna. 2 subfamilies (Callidosomatinae Southcott, 1961 and Abrolophinae Witte, 1995), 2 genera (Charletonia oudemans, 1910 and Abrolophus Berlese, 1891) and 1 species (Charletonia cardinalis (Pallas, 1772)) are new for the studied area. Abrolophus Berlese, 1891 genera are rich with species, 8 species have been found. The found species are spread over the altitudes and landscape zones, landscapes and forest biotopes. Also, the zoogeographic analysis of the erythraeid mites was carried out and they were divided into ecological groups. The studies show that the base of the fauna of the Erythraeidae Robineau-Desvoidy, 1828 mites in the Greater Caucasus region consists of 3 zoogeographic groups: European species (9 species), Central European species (5 species), Holarctic species (4 species).
\end{abstract}

Key words: Acari; Erythraeidae; Greater caucasus; Landscapes; Biotopes; Zoogeographic groups

\section{Introduction}

The fauna of the Caucasian Erythraeidae mites have not been studied almost carefully before our studies. Totally, 6 species belonging to 5 genera of 5 sub-families have been found: Eatoniana plumifera (Birula 1893), Leptus molochinus (C.L.Koch, 1837), Leptus species, Abrolophus species, Charletonia cardinalis, Myrmicothrombium species (Aslanov 2015; Beron 2008; Noei 2015; Makol and Sevsay 2015). The aim of our research is detailed study Erythraeid mites of the Greater Caucasus region of Azerbaijan. However, only 2 species of 2 genera (Leptus molochinus, Eatoniana plumifera) have been known in the Greater Caucasian part of Azerbaijan (Aslanov and Musayeva 2003). Today, 28 species of the Erythraeidae mites belonging to 9 genera of 6 sub-families are known in the Caucasian fauna thanks to our studies. As well as, 25 species belonging to 8 genera have been studied in the Greater Caucasian region. 1 sub-family (Balaustiinae), 4 genera (Erythraeus, Curteria, Balaustium, Neobalaustium) and 22 species are new for the Caucasian fauna. 2 sub-families (Callidosomatinae and Abrolophinae), 2 genera (Charletonia and Abrolophus) and 1 species (Charletonia cardinalis) are new for the studied area.

\section{Materials and Methods}

The Erythraeid mites are collected by different methods. The Erythraeidae mites are collected from the plant by means of shaking method. The Erythraeidae mites are collected by a fluffy thin brush soaked with water that fixes the mites from the walls of the buildings, trunks of the trees and bushes, the surface of soil and under the rocks. The collection of the Erythraeidae mites emerging in the soil, on the floor, in the autumn leaves, in the mosses and lichens, and on the tree trunks is carried out by means of eclector or sifter (Winkler device). Also, the hunting boxes (Barber traps) with fixed water are used for the collection of the Erythraeidae mites living in the soil. The $7 x-10 x$ hand lens is used for the collection of the Erythraeidae mites. The mites collected by different methods are included into the test tubes which have fixed water (70-75\% ethyl alcohol). Each test tube is labelled. In order to decolorize the mites, $50 \%$ lactic acid is used. The post-larval stage of the Erythraeidae mites is softened at $\mathrm{KOH}$ before the fixation. In order to define the mites and to study the morphological characteristic of them, the permanent drugs are made from these mites under the binocular (MBS-1 microscope) in the Phorate liquid (composition: $30 \mathrm{gr}$ hummiarabic, $200 \mathrm{gr}$ chloral- hydrate, 20 gr glycerine, $50 \mathrm{gr}$ water). The prescription of the preparations is carried out by means of suitable MBIS-3, Olympus CX-41, MBI15U4.2 microscopes. When working with the MBI-3 and MBI-15U4.2 microscopes, a phased contrast, oil or water immersion is used. The mites are measured by an ocular micrometre. The photos aretaken by means of RA- 6 painting apparatus or camera (Aslanov and Alizade 2018; Noei et al. 2019; Makol and Sevsay 2015).

The study was carried out in different localities of the Greater Caucasus natural region during 2017-2019 (Table 1 and Figure 1). 
Table 1. Localites and coordinates of sampling areas.

\begin{tabular}{|c|c|c|c|}
\hline \multicolumn{4}{|c|}{ Absheron peninsula } \\
\hline Guzdek Village & $40^{\circ} 22^{\prime} 24^{\prime \prime} \mathrm{N} / / 49^{\circ} 40^{\prime} 50^{\prime E}$ & Incirlik Village & $40^{\circ} 28^{\prime} 21^{\prime \prime} \mathrm{N} / / 50^{\circ} 5^{\prime} 44,8^{\prime \prime} \mathrm{E}$ \\
\hline Hokmeli Village & $40^{\circ} 25^{\prime} 51^{\prime \prime} \mathrm{N} / / 49^{\circ} 44^{\prime} 16^{\prime \prime} \mathrm{E}$ & Bayil forest & $40^{\circ} 21^{\prime} 0^{\prime \prime} \mathrm{N} / / 49^{\circ} 50^{\prime} 0^{\prime \prime} \mathrm{E}$ \\
\hline Gobu Village & $40^{\circ} 24^{\prime} 17^{\prime \prime} \mathrm{N} / / 49^{\circ} 42^{\prime} 45^{\prime \prime} \mathrm{E}$ & Badamdar plateau & $40^{\circ} 20^{\prime} 22^{\prime \prime} \mathrm{N} / / 49^{\circ} 48^{\prime} 14^{\prime \prime} \mathrm{E}$ \\
\hline $\begin{array}{l}\text { Lokbatan } \\
\text { Village }\end{array}$ & $40^{\circ} 19^{\prime} 38^{\prime \prime N} / / 49^{\circ} 43^{\prime} 48^{\prime \prime E}$ & Zabrat Settlement & $40^{\circ} 28^{\prime} 44^{\prime \prime} \mathrm{N} / / 49^{\circ} 56^{\prime} 52^{\prime \prime E}$ \\
\hline $\begin{array}{l}\text { Merdekan } \\
\text { Village }\end{array}$ & $40^{\circ} 29^{\prime} 32^{\prime \prime N} / / 50^{\circ} 8^{\prime} 20^{\prime \prime E}$ & $\begin{array}{l}\text { Jeyranbatan } \\
\text { Settlement }\end{array}$ & 40³2'24"N//49³9'34"E \\
\hline \multicolumn{4}{|c|}{ Gobustan district } \\
\hline Sundu Village & $40^{\circ} 19^{\prime} 22^{\prime \prime} \mathrm{N} / / 49^{\circ} 02^{\prime} 46^{\prime \prime} \mathrm{E}$ & Giceki mountain & $40^{\circ} 25^{\prime} 6^{\prime \prime N} / / 48^{\circ} 56^{\prime} 36^{\prime \prime E}$ \\
\hline Khilmilli Village & $40^{\circ} 41^{\prime} 31^{\prime \prime N} / / 48^{\circ} 51^{\prime} 49 " \mathrm{E}$ & Jeyrankechmez river & $40^{\circ} 29^{\prime} 19^{\prime \prime} \mathrm{N} / / 40^{\circ} 03^{\prime} 40^{\prime \prime} \mathrm{E}$ \\
\hline \multicolumn{4}{|c|}{ Shamakhi district } \\
\hline $\begin{array}{l}\text { Chuxuryurd } \\
\text { Village }\end{array}$ & $40^{\circ} 42^{\prime} 54^{\prime \prime N} / / 48^{\circ} 38^{\prime} 6^{\prime E}$ & Demirchi Village & $40^{\circ} 50^{\prime} 32^{\prime \prime} \mathrm{N} / / 48^{\circ} 33^{\prime} 45^{\prime \prime} \mathrm{E}$ \\
\hline $\begin{array}{l}\text { Dedegunesh } \\
\text { Village }\end{array}$ & $40^{\circ} 38^{\prime} 42^{\prime \prime N} / / 48^{\circ} 38^{\prime} 2^{\prime \prime E}$ & Gizmeydan Village & $40^{\circ} 46^{\prime} 11^{\prime \prime N} / / 48^{\circ} 44^{\prime} 52^{\prime \prime E}$ \\
\hline \multicolumn{4}{|l|}{ Khizi district } \\
\hline Bakhishli Village & $40^{\circ} 53^{\prime} 59^{\prime \prime N} / / 48^{\circ} 58^{\prime} 57^{\prime \prime E}$ & Altiaghac Village & $40^{\circ} 51^{\prime} 29^{\prime \prime} \mathrm{N} / / 48^{\circ} 56^{\prime} 10^{\prime \prime} \mathrm{E}$ \\
\hline $\begin{array}{l}\text { Saphbulaq } \\
\text { Village }\end{array}$ & $40^{\circ} 48^{\prime} 18^{\prime \prime N} / / 48^{\circ} 52^{\prime} 32^{\prime \prime E}$ & Aladash range & $40^{\circ} 51^{\prime} 59^{\prime \prime} \mathrm{N} / / 48^{\circ} 59^{\prime} 17 " \mathrm{E}$ \\
\hline Garabulaqchay V & llage & $40^{\circ} 50^{\prime} 0^{\prime \prime} \mathrm{N} / / 49^{\circ} 5^{\prime} 23^{\prime \prime} \mathrm{E}$ & \\
\hline \multicolumn{4}{|l|}{ Siyazan district } \\
\hline $\begin{array}{l}\text { Beshbarmaq } \\
\text { mountain }\end{array}$ & $40^{\circ} 57^{\prime} 30^{\prime \prime N} / / 49^{\circ} 13^{\prime} 56^{\prime \prime E}$ & Zarat Village & $40^{\circ} 56^{\prime} 42^{\prime \prime} \mathrm{N} / / 49^{\circ} 16^{\prime} 41^{\prime \prime} \mathrm{E}$ \\
\hline \multicolumn{4}{|l|}{ Shabran district } \\
\hline Erebler Village & $41^{\circ} 16^{\prime} 30^{\prime \prime N} / / 48^{\circ} 51^{\prime} 30^{\prime \prime E}$ & Devechi port lake & $41^{\circ} 20^{\prime} 39^{\prime \prime} \mathrm{N} / / 49^{\circ} 3^{\prime} 35^{\prime \prime} \mathrm{E}$ \\
\hline \multicolumn{4}{|c|}{ Khachmaz district } \\
\hline Lecet Village & $41^{\circ} 41^{\prime} 45^{\prime \prime} \mathrm{N} / / 48^{\circ} 37^{\prime} 17^{\prime \prime} \mathrm{E}$ & Murshudoba Village & $41^{\circ} 30^{\prime} 33^{\prime \prime} \mathrm{N} / / 48^{\circ} 45^{\prime} 22^{\prime \prime} \mathrm{E}$ \\
\hline \multicolumn{4}{|l|}{ Guba district } \\
\hline Ispik Village & $41^{\circ} 18^{\prime} 32^{\prime \prime N} / / 48^{\circ} 25^{\prime} 5^{\prime \prime E}$ & Susay Village & $41^{\circ} 18^{\prime} 40^{\prime \prime} \mathrm{N} / / 48^{\circ} 17^{\prime} 17^{\prime \prime E}$ \\
\hline Jimi Village & $41^{\circ} 3^{\prime} 21 " \mathrm{~N} / / 48^{\circ} 21^{\prime} 55^{\prime \prime} \mathrm{E}$ & Uzunmeshe Village & $41^{\circ} 21^{\prime} 35^{\prime \prime} \mathrm{N} / / 48^{\circ} 21^{\prime} 55^{\prime \prime E}$ \\
\hline \multicolumn{4}{|l|}{ Gusar district } \\
\hline Kuzun Village & & $41^{\circ} 20^{\prime} 27^{\prime \prime} N / / 48^{\circ} 8^{\prime} 9^{\prime \prime} E$ & \\
\hline \multicolumn{4}{|l|}{ Ismayilli district } \\
\hline Qalaciq Village & $40^{\circ} 57^{\prime} 33^{\prime \prime N} / / 48^{\circ} 3^{\prime} 9^{\prime \prime E}$ & Basqal Settlement & $40^{\circ} 45^{\prime} 20^{\prime \prime} \mathrm{N} / / 48^{\circ} 23^{\prime} 47^{\prime \prime} \mathrm{E}$ \\
\hline Tircan Village & $40^{\circ} 44^{\prime} 20^{\prime \prime} \mathrm{N} / / 48^{\circ} 20^{\prime} 1 " \mathrm{E}$ & Lahij Village & $40^{\circ} 50^{\prime} 57^{\prime \prime} \mathrm{N} / / 48^{\circ} 23^{\prime} 1,4^{\prime \prime} \mathrm{E}$ \\
\hline \multicolumn{4}{|l|}{ Gabala district } \\
\hline Hemzeli Village & $41^{\circ} 0 ' 9^{\prime \prime} \mathrm{N} / / 47^{\circ} 48^{\prime} 19^{\prime \prime} \mathrm{E}$ & Qemervan & $41^{\circ} 3^{\prime} 47^{\prime \prime N} / / 47^{\circ} 47^{\prime} 31^{\prime \prime E}$ \\
\hline \multicolumn{4}{|l|}{ Sheki district } \\
\hline Baş Layski & $41^{\circ} 21^{\prime} 3 " N / / 47^{\circ} 5^{\prime} 8^{\prime \prime E}$ & Bash Goynuk Village & $41,33^{\circ} 0^{\prime} 0^{\prime \prime} \mathrm{N} / / 47,12^{\circ} 0^{\prime} 0^{\prime \prime} \mathrm{E}$ \\
\hline \multicolumn{2}{|c|}{ Bash Shabalıd Village } & $41^{\circ} 18^{\prime} 12^{\prime \prime N} / / 47^{\circ} 7^{\prime} 11^{\prime \prime E}$ & \\
\hline \multicolumn{4}{|l|}{ Gakh district } \\
\hline Ilisu Village & $41^{\circ} 28^{\prime} 0,72^{\prime \prime N} / / 47^{\circ} 03^{\prime} 31,5^{\prime \prime E}$ & Qum Village & $41^{\circ} 27^{\prime} 26^{\prime \prime} N / / 46^{\circ} 54^{\prime} 35^{\prime \prime E}$ \\
\hline Lekit Village & $41^{\circ} 29^{\prime} 03^{\prime \prime N} / / 46^{\circ} 50^{\prime} 38^{\prime \prime} E$ & Gashgachay Village & $41^{\circ} 32^{\prime} 34^{\prime \prime N} / / 47^{\circ} 02^{\prime} 33^{\prime \prime E}$ \\
\hline \multicolumn{4}{|c|}{ Zagatala district } \\
\hline Jar Village & $41^{\circ} 40^{\prime} 19^{\prime \prime} \mathrm{N} /{ }^{4} 46^{\circ} 41^{\prime} 14^{\prime \prime} \mathrm{E}$ & Perzivan Village & $41^{\circ} 34^{\prime} 9^{\prime \prime N} / / 46^{\circ} 42^{\prime} 15^{\prime \prime} \mathrm{E}$ \\
\hline \multicolumn{2}{|l|}{ Qazangul Village } & $41^{\circ} 35^{\prime} 0^{\prime \prime} \mathrm{N} / / 46^{\circ} 38^{\prime} 0^{\prime \prime} \mathrm{E}$ & \\
\hline \multicolumn{4}{|l|}{ Balakan district } \\
\hline \multicolumn{2}{|c|}{ Zagatala State Reserve } & $41^{\circ} 48^{\prime} 7 " N / / 46^{\circ} 37^{\prime} 40^{\prime \prime} E$ & \\
\hline
\end{tabular}




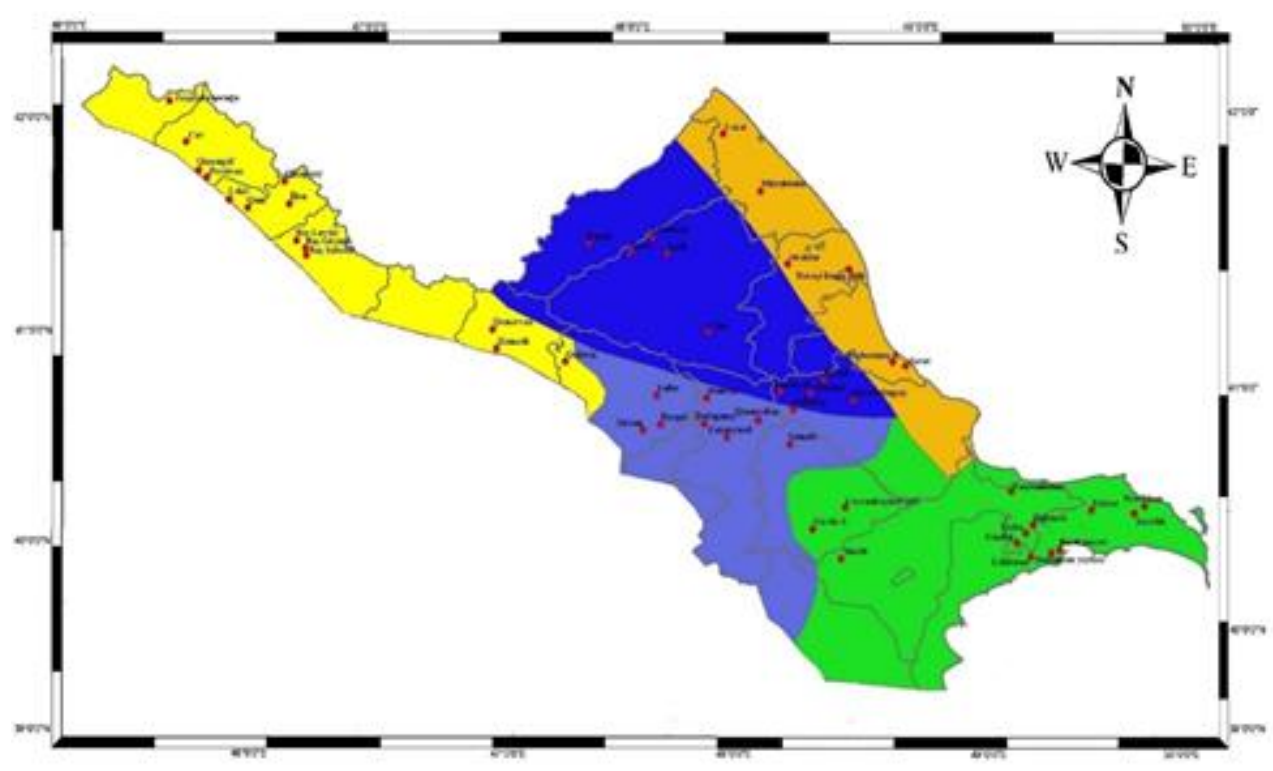

Figure 1. Map of study area.

\section{Results and Discussion}

\section{Erythraeidae}

Erythraeinae Robineau-Desvoidy, 1828; Erythraeus latreille, 1806; Erythraeus gorcensis, Gabrys 2016

Materials examined: Absheron peninsula, Baku, artificial forest, under the rocks, 20.V.1984 (1 f $\neq$ ) (collected by O.Aslanov); Lerik district, Gosmalian village 28.V.1985 (1 f₹) (collected by Kh.Aliyev).

Distribution: Central Europe, Poland, Switzerland (Beron 2008). The larvae and deutonymph were not found.

\section{Erythraeus phalangoides (De Geer, 1778)}

Materials examined: Absheron peninsula, Baku, artificial forest, under the rocks, 15. X.1984 (2 spp.) (collected by O. Aslanov).

Distribution: Europe; Caucasus: Azerbaijan; Asia: Kuril Islands; Greenland (Beron 2008) (Figure 2).

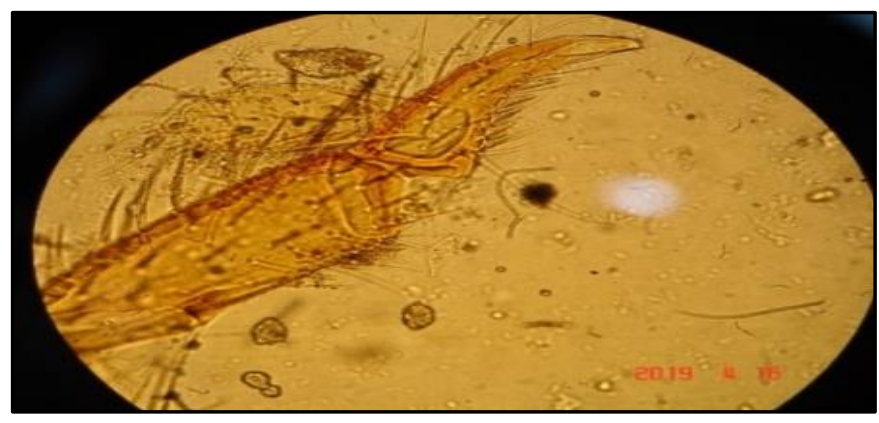

Figure 2. Pedipalp's genu and tibia of the Erythraeus phalangoides.

\section{Erythraeus regalis (C.L. Koch, 1837)}

Materials examined: Absheron peninsula, Baku, under the rocks, 20.V.1984 (3 spp.) (collected by O. Aslanov); Baku, Garden of the Institute of Zoology of ANAS, over the red-berry mite (Homoptera suborder), 18.V.1988 (1 larva) (collected by O. Aslanov); Gobustan region, Sundu village, different grassy steppe, plants, 15.V.2018 (1 spp.); Gakh district, Gum village, chestnut forest, plants, 29.VI.2018 (2 spp.).

Distribution: Europe; Caucasus: Azerbaijan; North-Western Africa: Algeria (Beron 2008), (Figure 3 ).
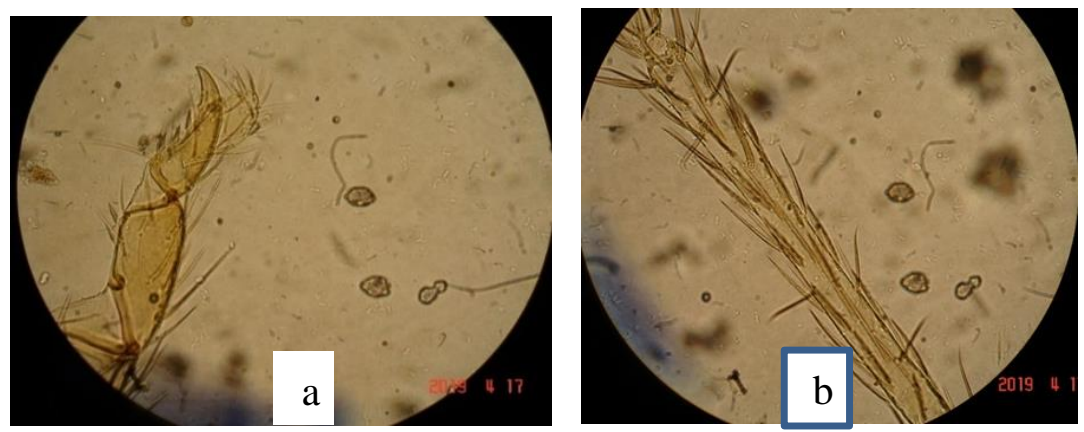

Figure 3. Erythraeus regalis. Pedipalp (tarsus, tibia and genu); b) tibia of the first leg. 
Erythraeus opilionoides (C.L. Koch, 1837)

Materials examined: Ismayilli district, Galacig village, broad-leaved forest, under the rocks, 22.VI.2014 (1 spp.) (collected by 0. Aslanov). Lerik district, Gosmalian village, 28.V.1985 ( $1 \mathrm{f} \neq$ ) (collected by Kh. Aliyev).

Distribution: Europe; Caucasus: Azerbaijan (Wohltmann 2013) (Figure 4).

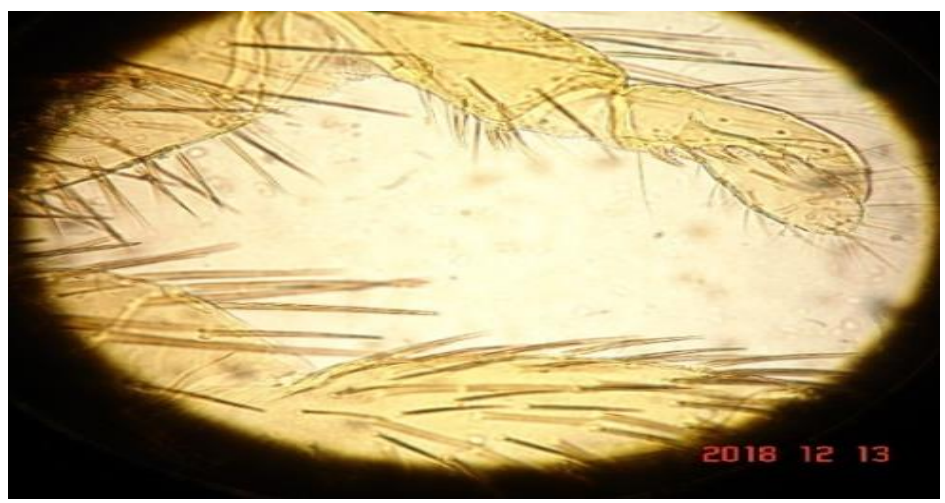

Figure 4. Pedipalp of the Erythraeus opilionoides.

Erythraeus adpendiculatus (Schrank, 1781)

Materials examined: Absheron peninsula, Baku, artificial forest, under the rocks, 12.V.1984, (1spp.) (collected by O. Aslanov); 20.V.1984 (5 spp.) (collected O. Aslanov) (Figures 5 and 6).

Distribution: Caucasus: Azerbaijan (Wohltmann 2013).

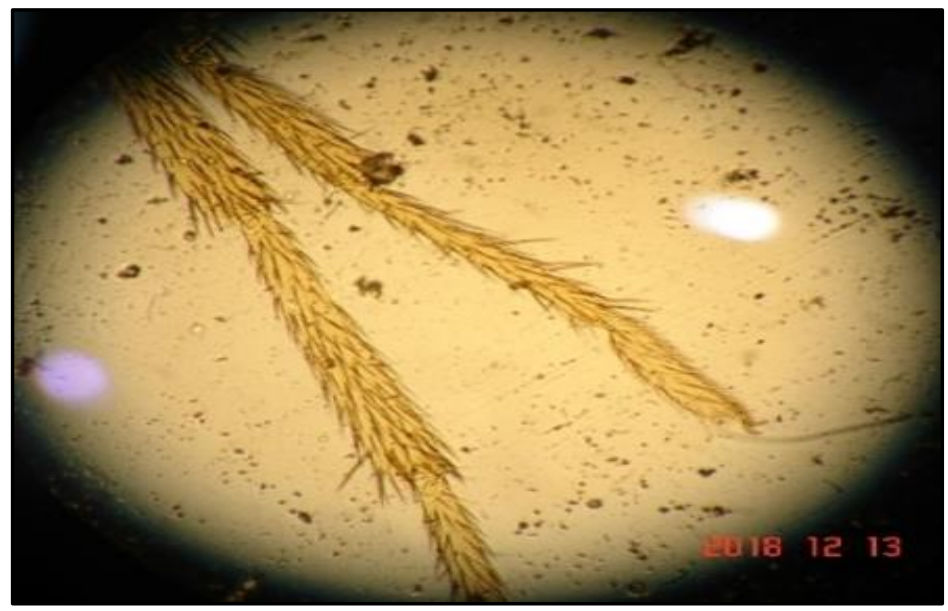

Figure 5. III and IV legs' genu, tibia and tarsus of Erythraeus adpendiculatus.
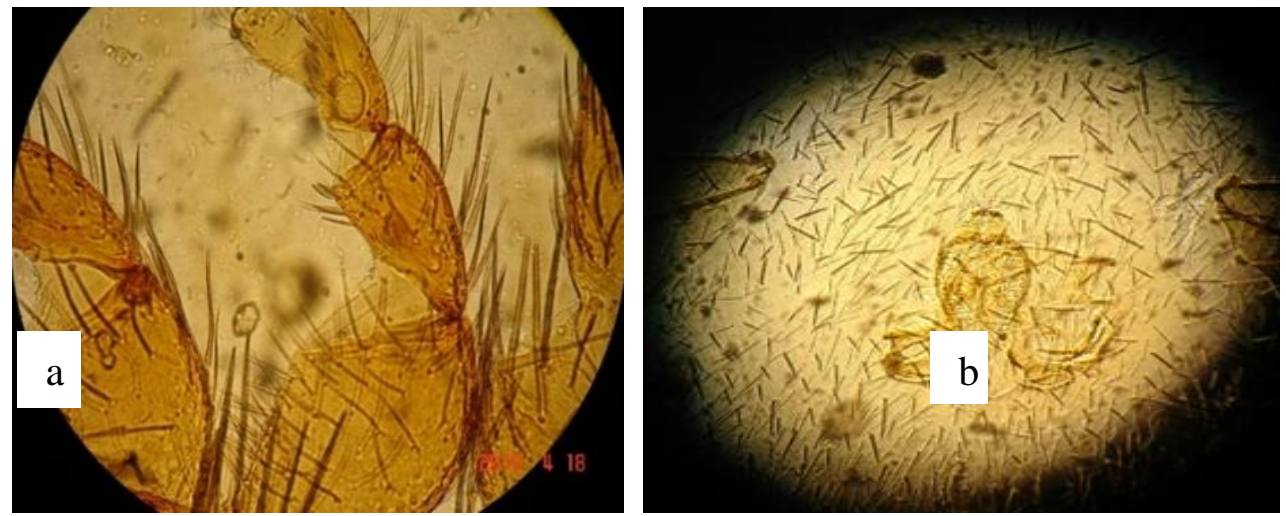

Figure 6. Erythraeus adpendiculatus. a) pedipalp; b) Genital apparatus of male.

Eatoniana Cambridge, 1898; Eatoniana plumifera (Birula, 1893)

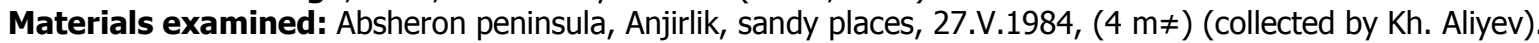

Distribution: The Southern Caucasus; Middle Asia: Turkmenistan (Beron 2008)

Eatoniana plumifera species are the indicator of the eol-sand landscape (Figure 7). 


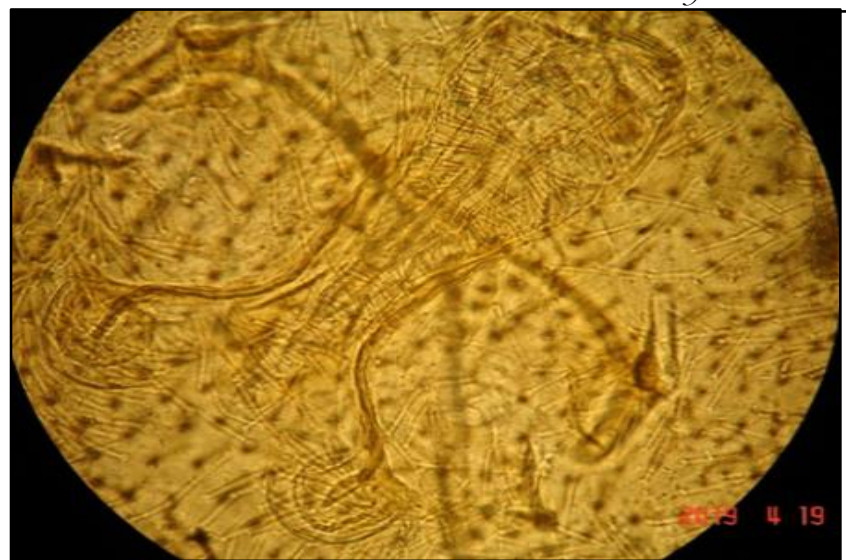

Figure 7. Genital apparatus of male of the Eatoniana plumifera.

Curteria; Curteria episcopalis (C.L. Koch, 1837)

Materials examined: Absheron peninsula, Anjirlik, sandy places, 1 adult individual, 27.V.1984 (1 spp.) (collected by Kh. Aliyev); Gakh district, Gashgachay village, middle mountain haw-peanuts forest, plants, 30.VI.2018 (1 larva).

Distribution: Central (Germany, Netherland and Poland) and Northern Europe (Switzerland and Finland); Asia: Iran (Jamshidian et al. 2014 (Figure 8).

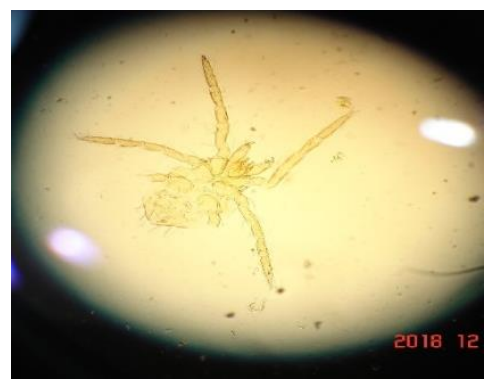

Figure 8. General view of the Curteria episcopalis.

Leptinae, Southcott, 1957; Leptus Latreille, 1796; Leptus slivovi Beron, 1975

Materials examined: Guba district, Jimi village, oak-peanut forest (with Caucasian oak), trunks of tree, $29 . \mathrm{VI} .990$ (1 larva) (collected by O. Aslanov); Guba district, Jimi village, oak forest (with Caucasian oak), plants, 5. VIII.2018 (1 larva); Guba district, Susay village, Gizilaghaj forest, harvestman (Opilio species), 4.VIII.2018 (1 larvae and 2 nymphochrysalis (pup I)); Shabran district, Arablar village, mountainous forest-desert, plants, 9.V.2018 (1 larva); Gobustan region, Chalov village, steppe, grasses, $17 . V .2019$ (1 larva) (Figure 9).
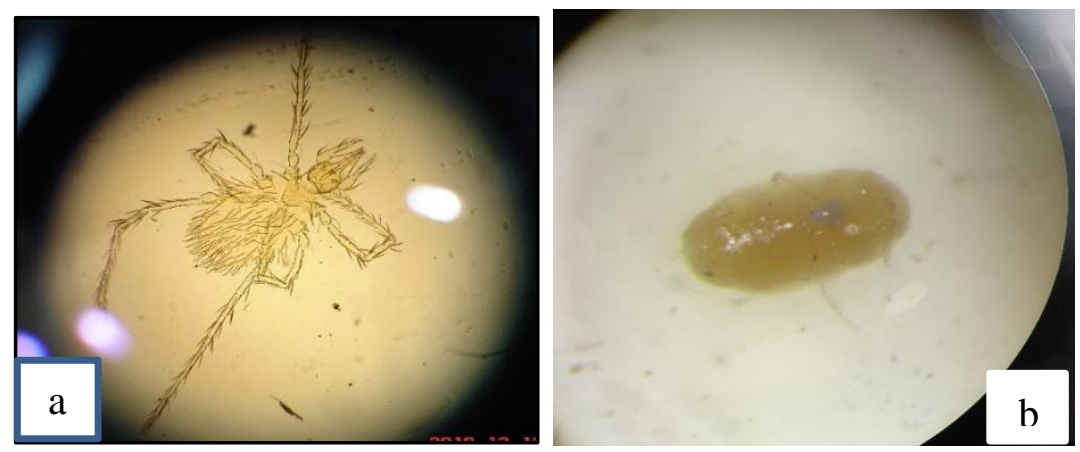

Figure 9. Leptus slivovi: a) General view of larva; b) Nymphochrysalis.

Distribution: Northern (Norway), Central (Poland, Liechtenstein) and Southern (Bulgaria) Europe; Southern Caucasus (Azerbaijan); Asia: South-Eastern Kazakhstan (Aslanov 2015; Beron 2008).

Leptus clethrionomydis Haitlinger, 1987

Materials examined: Zagatala district, ether-oil sovkhoz plant, tea plantation, tea bush, 12.VIII.2017 (1 larva).

Distribution: Central Europe: Poland (Clethrionomys glareolusda) was found in European striped field mouse), Hungary; Southern Caucasus: Azerbaijan (Aslanov and Alizade 2018; Beron 2008).

Leptus molochinus (C.L. Koch, 1837)

Materials examined: Khachmaz district, Murshudoba village, fruit garden, on the foot of harvestman, 15. VIII.1985 (1larva) (collected by Z. Musayeva); Guba district, Ispik village, peanut forest, moss, 5.VIII.2018 (1 larva); Guba district, Uzunmeshe village, alder forest (with grey alder tree), plants, 4.VIII.2018 (1 larva); Siyazan region, Galaalti village, oak forest (with Georgian oak trees), plants, 25.V.2019 (1 larva).

Distribution: Europe; Caucasus: Azerbaijan; Northern America: USA, Greenland Island (Beron 2008) (Figure 10). 


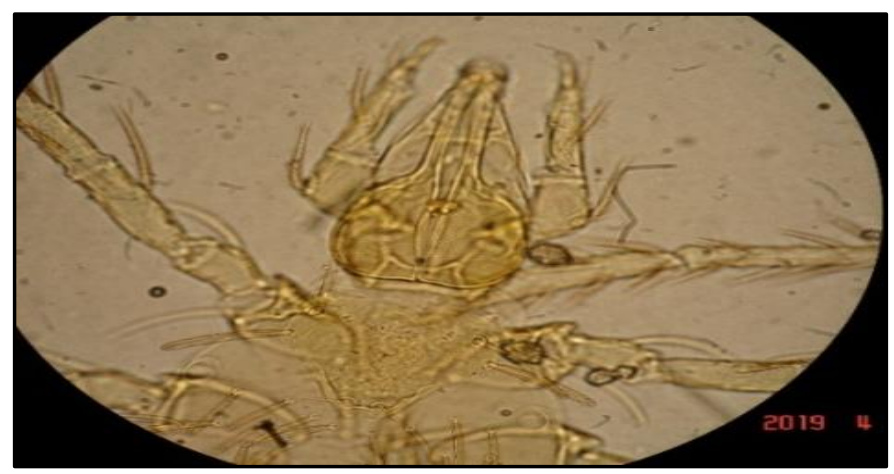

Figure 10. Scutum of the larva of the Leptus molochinus.

Leptus Iongipilis (Berlese, 1910)

Materials examined: Zagatala district, Parzivan village, mountain broad-leaved forest, floor, II.1986 (1 spp.) (collected by I. Kulagina).

Distribution: Central (Poland and Hungary) and Southern (Italy) Europe; Southern Caucasus (Azerbaijan) (Beron, 2008)

Comments: Leptus longipilis species are an indicator of the landscape of broad-leaved forests of the mountain-forest zone and lowmountains

Leptus rubricatus (C.L. Koch, 1837)

Materials examined: Zagatala district, Parzivan village, mountain broad-leaved forest, II.1986 (1 spp.) (collected by I. Kulagina); Zagatala district, Parzivan village, mountain broad-leaved forest, floor, IV.1986 (1 spp.) (collected by İ.Kulagina); Gakh district, Ilisu village, broad-leaved forest, under the stones, 5.V.2013 (1 spp.) (collected by O.Aslanov); Balakan district, Zagatala preserve, mountain broad-leaved forest, soil decoy, 2.V.2015 (1 spp.) (collected by N.Sneqovaya) (Figure 11).

Distribution: Europe; Caucasus: Azerbaijan (Beron 2008)

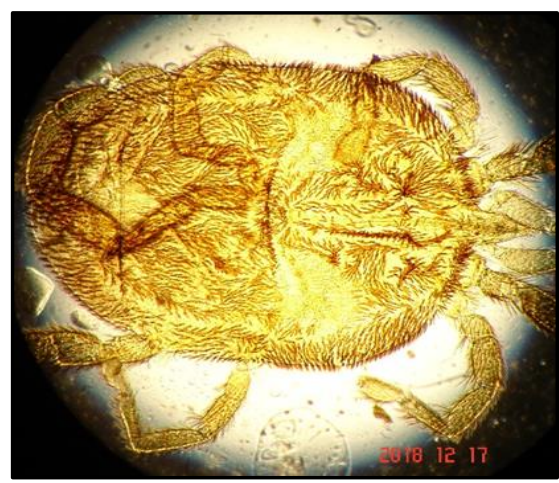

Figure 11. General view of the Leptus rubricatus.

Callidosomatinae; Charletonia; Charletonia globigera (Berlese, 1885)

\section{Materials examined}

Lankaran district, in the forest floor, IX.1984 (2 spp.) (collected by Kh. Aliyev). Siyazan district, Zarat settlement, Gangiz desert, 26.V.2017 (9 spp.); Khizi district, Garabulagchay, slippery fields, plants, 26.VIII.2017 (2 spp.); Khizi district, Bakishli village, thorn shiblyak, thorn, 26.VIII.2017 (2 spp.) ; Shabran district, Arablar village, forest-steppe, plants, $8 . V .2018$ (1 f $\neq$ ); Siyazan district, Beshbarmaq mountain, shoranga desert, shoranga, 15.IX.2018 (8 spp.); Khizi district, Bakhishli village, arid spruce forest consisting of dwarf pear trees, a dwarf pear, 1.VI.2018 (1 sp.); Ismayilli district, Tirjan village, birch-oak forest (with marvellous birch), 2.IX.2018 (5 spp.); Gakh district, Lakit village, oak forest (with Georgian oak tree), plants, $29 . V I .2018$ (1 f $\neq$ ); Zagatala district, Jar village, hornbeam forest, plants, 30.VI.2018 (1 sp.).

Distribution: Europe; Southern Caucasus (Azerbaijan) (Beron 2008)

Charletonia cardinalis, Pallas 1772

Materials examined: Siyazan district, Beshbarmaq Mountain, shoranga desert, shoranga, $15 . \mathrm{IX} .2018$ (1 sp.); Babak district, Garachuq village, inside the wheat, ( 2 larvae) (collected by E.Abdullayeva); Sabirabad district, over the striped bee, VI.1972 (5 larvae) (collected by Sokolova).

Distribution: is cosmopolit species (Haitlinger 1987) (Figure 12). 


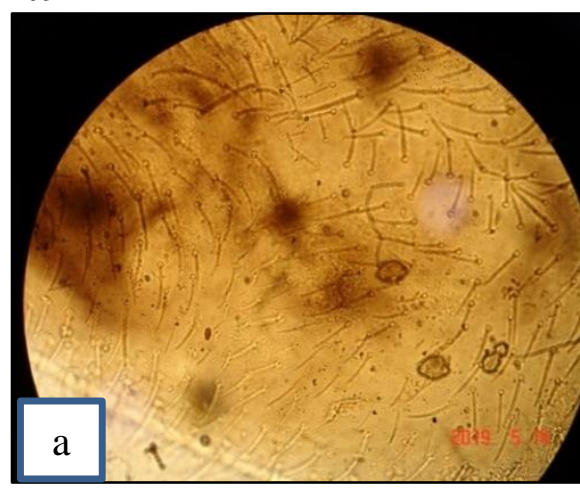

Figure 12. Charletonia cardinalis. a) Dorsal opistosomal setae of adult; b) Larva's gnatosom.

Abrolophinae; Abrolophus; Abrolophus artemisiae (Schrank, 1803)

Materials examined: Absheron peninsula, Baku city, artificial-forest massive, floor of the pine tree, $31 . \mathrm{V} .1986$ (1 sp.) (collected by O. Aslanov).

Distribution: Europe; Southern Caucasus (Azerbaijan) (Beron 2008)

Abrolophus miniatus (Hermann, 1804)

Materials examined: Khizi district, Garabulagchay, slippery fields, plants, 26.VIII.2017 (1 f $\neq$ ); Khizi district, Bakhishli village, thorn shiblyak, thorn, 26.VIII.2017 (3 spp.); Khizi district, Bakhishli village, oak forest (with Georgian oak tree), plants, 1. VI.2018 (1 sp.); Gakh district, Saribash village, field-bush, plants, 29.VI.2018 (1 sp.); Gakh district, Gashgachay village, hornbeam-beech forest, plants, 30.VI.2018 (2 f¥); Guba district, Uzunmeshe village, alder-hornbeam forest, plants, 4.VIII.2018 (1 sp.); Guba district, Ispik village, beech forest, moss, 5.VIII.2018 $(1 \mathrm{f} \neq)$; Khizi district, Aladash chain, forest-steppe, plants, $15 . I X .2018(1 \mathrm{f} \neq)$; Gabala district, Gamarvan village, hornbeam forest, plants, 30.IX.2018 (1 sp.).

Distribution: Europe; Caucasus: Azerbaijan; North-Western Africa (Algeria); Greenland Island (Beron 2008)

\section{Abrolophus crassitarsus (Schweizer, 1951)}

Materials examined: Zagatala district, ether-oil sovkhoz-plant, tea plantation, tea bush, 12. VIII.2017 (1 sp.); Khizi district, Bakhishli village, oak forest (with Georgian oak tree), grasses, 26.VIII.2017 (1 sp.); Khizi district, Bakhishli village, oak forest, plants, 26.VIII.2017 (1 $\mathrm{m \neq}$ ); Khizi district, Bakhishli village, palıd forest, tree corona, 26. VIII.2017 (5 spp.); Khachmaz district, Lajat village, plain forest, plants, 9.V.2018 (3 spp.); Khizi district, Bakhishli village, arid spruce forest (with dwarf pear trees), $1 . V I .2018$ (2 spp.); Gakh district, Saribash village, field-bush, 29.VI.2018 (1 sp.); Guba district, Jimi village, oak forest (with eastern oak), plants, 5.VIII.2018 (5 spp.); Ismayilli district, Lahij village, forest, plants, 2.IX.2018 (3 spp.); Ismayilli district, Basgal village, hornbeam forest, plants, 2.IX.2018 (2 spp.); Ismayilli district, Lahij village, forest, plants, 2.IX.2018 (3 spp.); Shabran district, Arablar village, semi-desert, plants, 25.V.2019 (2 spp.); Shamakhi district, Dadagunash village, oak forest (with Georgian oak tree), plants, 17.V.2019 (11 spp.); Shamakhi district, Chukhuryurd village, arid spruce forest (consisting of dwarf pear), plants, $17 . V I .2019$ (2 spp.).

Distribution: Central Europe; Southern Caucasus (Azerbaijan) (Beron 2008) (Figure 13).

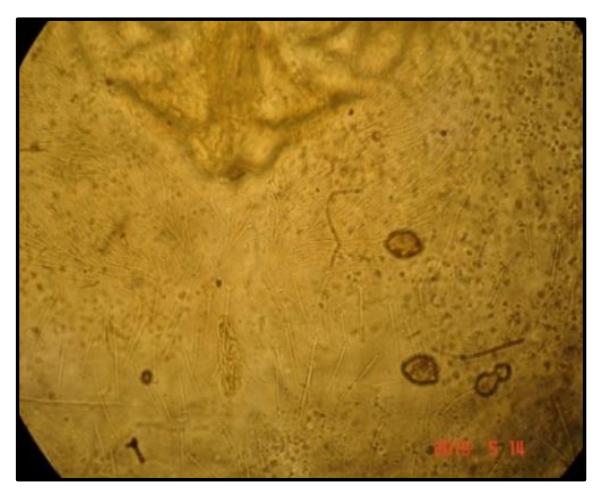

Figure 13. Anus of the Abrolophus crassitarsus.

Abrolophus rhopalicus (C.L. Koch, 1837)

Materials examined: Shamakhi district, Chukhuryurd village, field-steppe, plants, 15.V.2018 (3 f $\neq$ ).

Distribution: Europe; Southern Caucasus (Azerbaijan) (Beron 2008).

Comments: Abrolophus rhopalicus is an indicator of mountain forest-steppe landscape.

Abrolophus passerinii (Berlese, 1904)

Materials examined: Absheron district, Jeyranbatan settlement, artificial forest massive, mint, $20 . \mathrm{VI} .1985$ (1 sp.) (collected O. Aslanov); Khizi district, Altiaghac settlement, forest, grass, 26.V.2011 (1 sp., 3 larvae) (collected by O.Aslanov).

Distribution: Ireland Island, Central (Poland) and Southern (Italy) Europe; Southern Caucasus (Azerbaijan) (Beron, 2008) 


\section{Abrolophus strojnyi Gabrys, 1992}

Materials examined: Khizi district, Yarimja village, oak forest (with Georgian oak tree), plants, $20 . V I .2012$ (1 sp.) (collected by O. Aslanov); Gakh district, Ilisu village, juniper-keeping, juniper, 5.V.2013 (3 spp.) (collected by O. Aslanov); Zagatala district, Jar village, hornbeam forest, plants, 30.VI.2018 (4 spp.).

Distribution: Central Europe (Poland), Hungary; Southern Caucasus (Azerbaijan) (Beron 2008).

Comments: Abrolophus strojnyi is an indicator of montane forest zone (Figure 14).

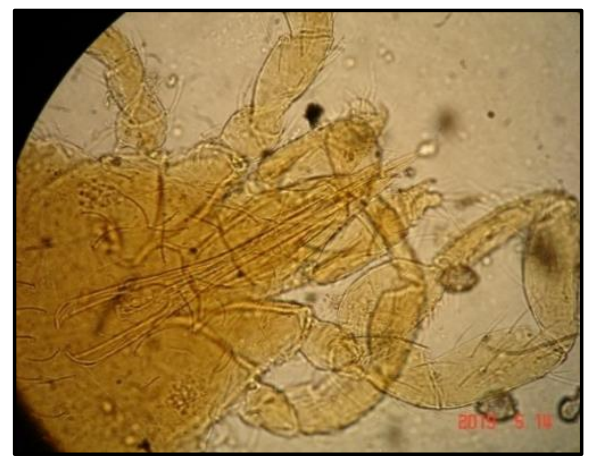

Figure 14. Crista metopica of the adult of the Abrolophus strojnyi.

Abrolophus quisquiliarus (Hermann, 1804)

Materials examined: Absheron peninsula, Baku city, rocks, 10.V.1985 (1 q ) (collected by O.Aslanov); Khizi district, Bakhishli village, oak forest (with Georgian oak tree), 1.VI.2018 (4 spp.); Khizi district, Bakhishli village, arid spruce forest (consisting of dwarf pear trees), a dwarf pear, 1.VI.2018 (1 sp.). Lerik district, Gosmalian village, under the rocks, 28. VI.1985 (1 sp.) (collected by Kh. Aliyev).

Distribution: Europe; Caucasus: Azerbaijan (Beron 2008)

Comments: Abrolophus quisquiliarus is an indicator of mountainous-forest zone.

Abrolophus norvegicus (Thor, 1900)

Materials examined: Gusar district, Kuzun village, sub-alp birch forest, Radde birch, 4.VIII.2018 (4 spp.); Ismayilli district, Tirjan village, birch forest (including marvelous birch), plants, 2.IX.2018 (1 sp.). Shamkir district, Ashagi Seyfali village, in the black clover field, 25.VIII.1985 (1 sp.) (collected by O. Aslanov).

Distribution: Europe; Caucasus: Azerbaijan (Beron 2008).

Comments: Abrolophus norvegicus species are the effective regulator of the number of the sucking pests of the black clover in the black-clover fields of Shamkir district. Also, these species are an indicator of mountainous-forest zone.

Balaustiinae; Balaustium; Balaustium xerothermicum Gabrys, 2000

\section{Materials examined}

Zagatala district, Parzivan village, mountain broad-leaved forest, floor, IV.1986 (2 spp.) (collected by I. Kulagina); Zagatala district, tea plantation, 7.VII.2019 (1 sp.).

Distribution: Central Europe (Poland); Southern Caucasus (Azerbaijan) (Beron 2008) (Figure 15).

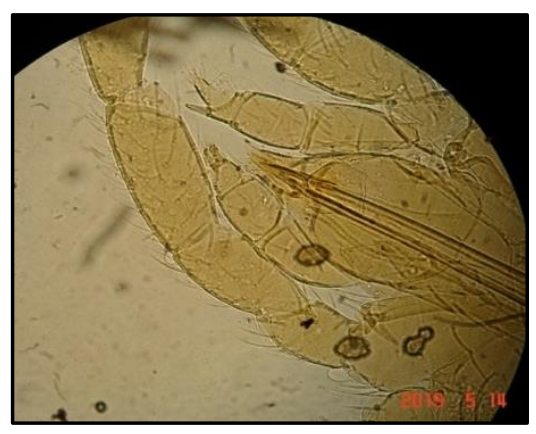

Figure 15. Gnathosoma of the Balaustium xerothermicum.

Balaustium unidentatum (Tragardh, 1904)

Materials examined: Absheron peninsula, Mardakan settlement, over the pool, 19.V.1985 (6 spp.) (collected by O. Aslanov); Baku city, Garden of the Institute of Zoology of ANAS, Azadirachta indica, 19.V.1988 (1 sp.) (collected by O. Aslanov); Shamakhi district, Chuxuryurd village, forest- steppe, plants, 15.V.2018 (2 spp.) (Figure 16). 

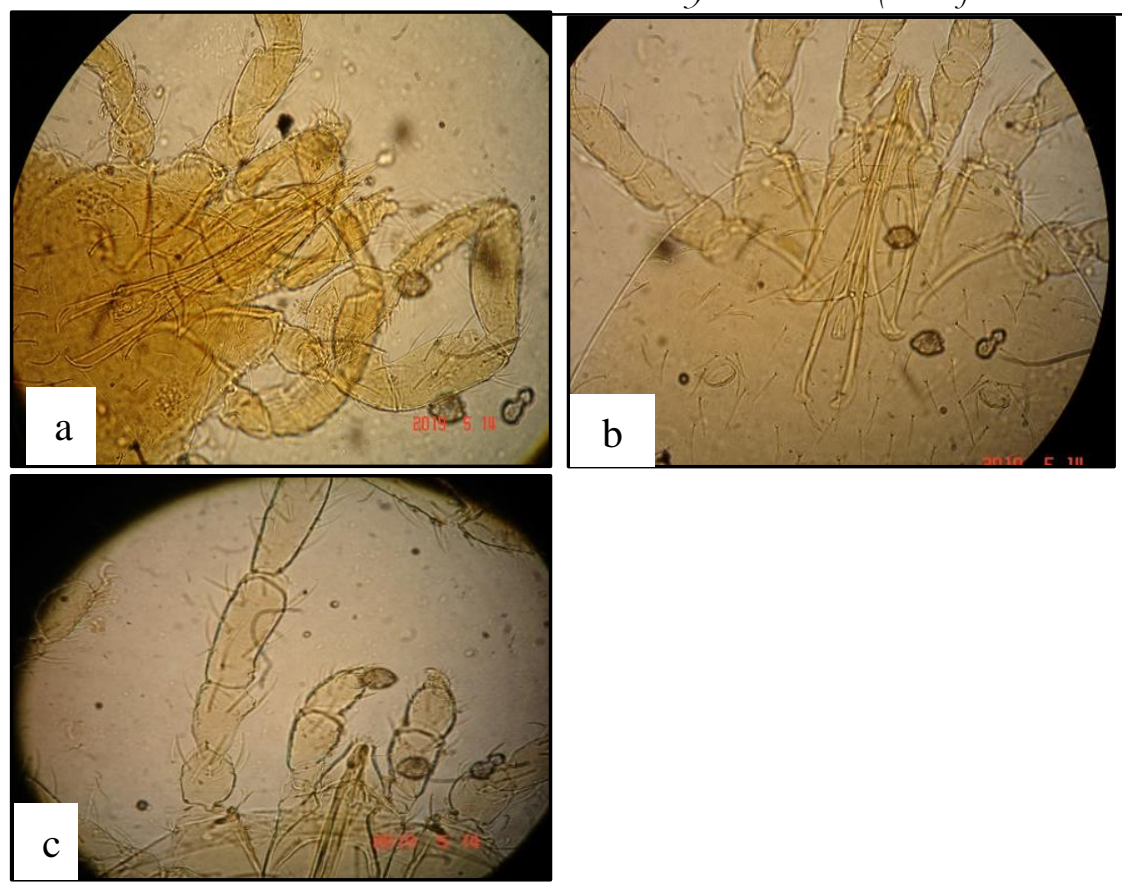

Figure 16. Balaustium unidentatum: a) general view; b) urnulae and eyes; c) pedipalp.

Distribution: Central Europe (Poland and Switzerland); Caucasus: Azerbaijan; Greenland Island (Beron 2008)

Comments: Female individuals come into surface massively in order to lay an egg in the second half of May. In addition, at that time, the surface of soil and rocks are seen dark red.

\section{Neobalaustium; Neobalaustium species}

Materials examined: Gakh district, Ilisu village, mountain broad-leaved forest, under the rocks, 5.V.2013, (3 spp.) (collected by O.Aslanov); Khizi district, Garabulaqchay, slippery fields, plants, 25.VIII.2017 (2 spp.); Siyazan district, Zarat settlement, shoranga desert, shoranga, 25.VIII.2017 (1 sp.); Siyazan district, Beshbarmaq mountain, Gangiz desert, Gangiz Village 25. VIII.2017 (31 spp.); Shamakhi district, Chuxuryurd village, forest-steppe, plants, 15.V.2018 (2 spp.); Siyazan district, Beshbarmaq mountain, shoranga desert, shoranga, 15.IX.2018 (1 f $\neq$ ); Shabran district, Arablar village, forest-steppe, plants, $25 . V .2019$ (3 spp., 3 larvae); Siyazan district, Beshbarmaq mountain, arid spruce forest (consisting of dwarf pear trees), over the rocks, $25 . \mathrm{V} .2019$ (5 $\mathrm{f} \neq$ ); Gobustan district, Chalov village, steppe, grasses, 17.V.2019 (5 spp.).

Comments: Female individuals come into surface massively in order to lay an egg in the second half of May, and at that time, the surface of soil and rocks are seen dark red. 6 eggs increase in the body of the female individuals at the same time.

During our research we investigated distribution of erythraeid mites of the Greater Caucasus by landscape belts (Table 2), by altitudinal zonation (Table 3), by landscapes (Table 4) and by forest biotopes (Table 5), too.

Table 2. Distribution of erythraeid mites of the Greater Caucasus by landscape belts.

\section{Species}

Erythraeus gorcensis

Erythraeus opilionoides

Erythraeus phalangoides

Erythraeus adpendiculatus

Erythraeus regalis

Curteria episcopalis

Neobalaustium species

Eatonia plumifera

Leptus rubricatus

Leptus longipilis

Leptus slivovi

Leptus molochinus

Leptus clethrionomydis

Abrolophus artemisae

Abrolophus miniatus

Abrolophus crassitarsus

Abrolophus rhopalicus

Abrolophus passerinii

Abrolophus strojnyi

\begin{tabular}{|c|c|c|c|}
\hline \multicolumn{4}{|c|}{ Landscape belts } \\
\hline \multirow[t]{19}{*}{ A } & B & C & D \\
\hline & & & + \\
\hline & + & & \\
\hline & & & + \\
\hline & & & + \\
\hline & + & + & \\
\hline & + & & + \\
\hline & + & + & + \\
\hline & & & + \\
\hline & + & & + \\
\hline & + & & \\
\hline & + & + & \\
\hline & + & & + \\
\hline & + & & \\
\hline & & & + \\
\hline & + & + & + \\
\hline & + & + & + \\
\hline & & + & \\
\hline & & + & + \\
\hline & + & & \\
\hline
\end{tabular}




\begin{tabular}{lccc}
\hline Abrolophus norvegicus & + & + & \\
Abrolophus quisquiliarum & & + & \\
$\begin{array}{l}\text { Charletonia globigera } \\
\text { Charletonia cardinalis }\end{array}$ & + & + & + \\
$\begin{array}{l}\text { Balaustium xerothermicum } \\
\text { Balaustium unidentatum }\end{array}$ & & & + \\
\hline
\end{tabular}

Note: A - Subalpine zone; B - Montane forest; C - Alpine meadow; D - Semidesert.

Table 3. Distribution of erythraeid mites of the Greater Caucasus by altitudinal zonation.

\begin{tabular}{|c|c|c|c|c|c|}
\hline \multirow{2}{*}{ Species } & \multicolumn{5}{|c|}{ Altitudinal zonation } \\
\hline & $\mathbf{A}$ & B & C & D & $\mathbf{E}$ \\
\hline Erythraeus gorcensis & + & & & & \\
\hline Erythraeus opilionoides & & & + & & \\
\hline Erythraeus phalangoides & + & & & & \\
\hline Erythraeus adpendiculatus & + & & & & \\
\hline Erythraeus regalis & & & + & & \\
\hline Curteria episcopalis & + & & + & + & \\
\hline Neobalaustium species & & + & + & + & \\
\hline Eatonia plumifera & + & & & & \\
\hline Leptus rubricatus & + & & + & + & \\
\hline Leptus longipilis & & & + & & \\
\hline Leptus slivovi & & + & & + & \\
\hline Leptus molochinus & + & & & + & \\
\hline Leptus clethrionomydis & & + & & & \\
\hline Abrolophus artemisae & + & & & & \\
\hline Abrolophus miniatus & & + & + & + & \\
\hline Abrolophus crassitarsus & & + & + & + & \\
\hline Abrolophus rhopalicus & & & + & & \\
\hline Abrolophus passerinii & + & & + & & \\
\hline Abrolophus strojnyi & & & + & + & \\
\hline Abrolophus norvegicus & & & + & & + \\
\hline Abrolophus quisquiliarum & & & + & & \\
\hline Charletonia globigera & & + & + & & \\
\hline Charletonia Cardinalis & & & + & & \\
\hline Balaustium xerothermicum & & & + & & \\
\hline Balaustium unidentatum & + & & & & \\
\hline
\end{tabular}

Note: A - Lowland zone (-28-200 m); B - Foothill zone (200-500 m); C - Thermo-mediterranean zone (500-1000 m); D - Mesomediterranean zone (1000-2000 m); E - Super-mediterranean zone (>2000 m)

Table 4. Distribution of erythraeid mites of the Greater Caucasus by landscapes.

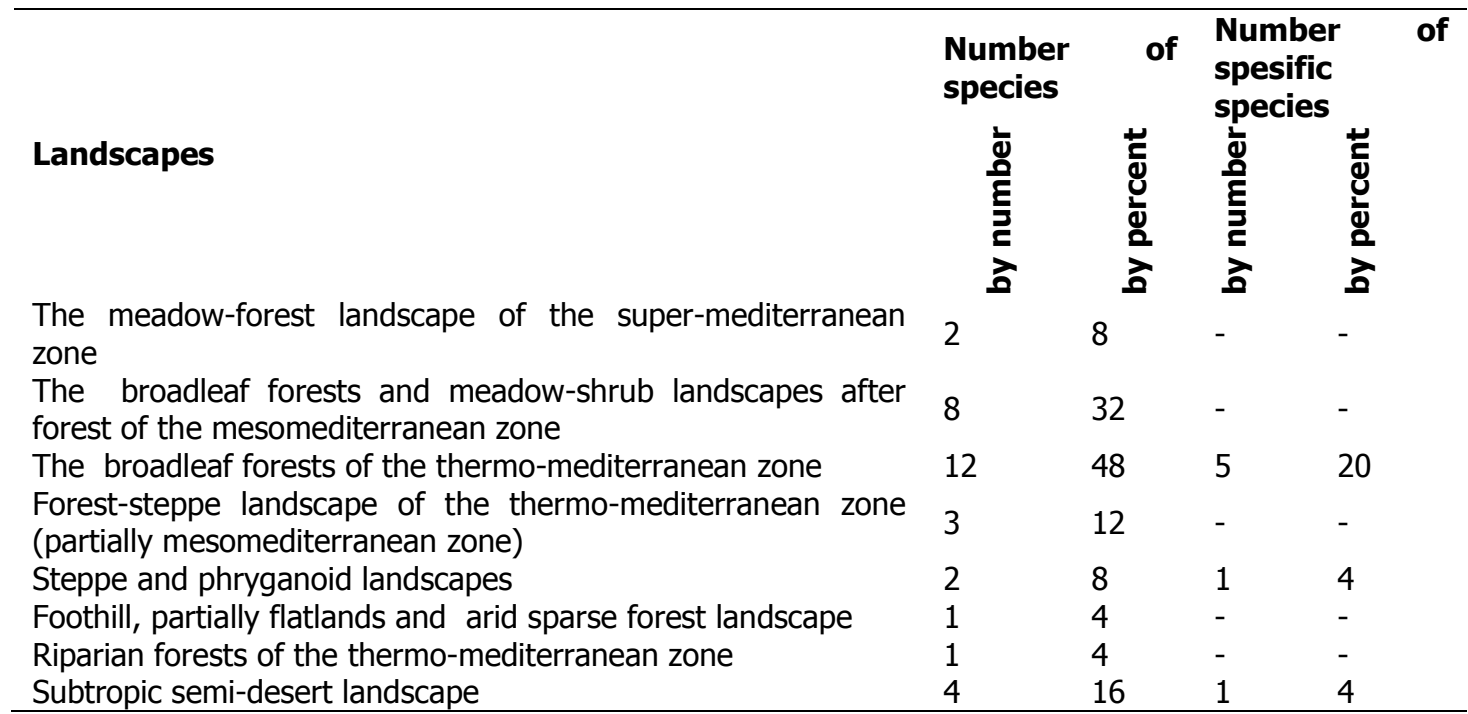




$\begin{array}{lllll}\text { Eol-sand landscape of the lowland zone } & 2 & 8 & 1 & 4 \\ \text { Artificial forests } & 6 & 24 & 4 & 16 \\ \text { Agro-landscapes } & 2 & 8 & 1 & 4\end{array}$

Table 5. Distribution of erythraeid mites of the Greater Caucasus by forest biotopes.

\section{Biotopes}

The hornbeam forests of thermo-mediterranean zone

The chestnut forests of thermo-mediterranean zone

The oak forests of thermo-mediterranean zone (with Quercus iberica)

The maple forests of thermo-mediterranean zone

The beech and beech-hornbeam forests of mesomediterranean zone

The oak and oak-beech forests of mesomediterranean zone

The Alnus forests of mesomediterranean zone (with Alnus incana)

The birch forests of super-mediterranean zone (with Betula raddeana)

The Tuqay forests

$\begin{array}{ll}\begin{array}{l}\text { Number of species } \\ \text { by } \\ \text { numbers } \\ 7\end{array} & \begin{array}{l}\text { by } \\ \text { percent } \\ 28\end{array} \\ 1 & 4 \\ 4 & 16 \\ 2 & 8 \\ 3 & 12 \\ 2 & 8 \\ 2 & 8 \\ 2 & 8 \\ 1 & 4\end{array}$

\section{Conclusion}

25 species of the erythraeid mites belonging to 8 genera of 5 sub-families in the Greater Caucasus region were found. Among them, 1 sub-family (Balaustiinae), 4 genera (Curteria, Erythraeus, Balaustium and Neobalaustium) and 22 genera are new for the Caucasian fauna. 2 sub-families, 2 genera and 1 species are new for the studied field. The most of the species are found as 10 species belonging to 5 genera in Absheron peninsula and 8 species belonging to 6 genera in Gakh district.

\section{Acknowledgments}

I express my gratitude to Oktay Aslanov from Institute of Zoology, NAS of Azerbaijan Republic for identification of collected mite species.

\section{References}

Aslanov, O., Musayeva, Z. (2003). To study of Acarofauna of Azerbaijan. Materials of the Ith congress of Azerbaijan Society of Zoologists. Azerbaijan, Baku: pp.144-146 (In Azeri with English abstract).

Aslanov, O. (2008). To study of predatoty Actinedid mites (Acariformes: Actinedida) of Azerbaijan. Proceedings of the Azerbaijan Society of Zoologists, vol. I: 210-216 (In Russian with English abstract).

Aslanov, O. (2015). The First Record of Leptus slivovi (Acariformes: Erythraeidae) in Kazakhstan. Vestnik Zoologii, 49 (3): 285 (In Russian with English abstract).

Aslanov, O., Rubtsova L., Khanbekova, Y. (2015). Mites, associated with honeybees (Aphis mellifera caucasica Gorb.) in Azerbaijan. Proceedings of the Azerbaijan Society of Zoologists, 7(2): 119-126

Aslanov, O. (2015). To study of Actinedid mites (Acariformes: Actinedida) of Azerbaijan. Proceeding of the Azerbaijan Institute of Zoology, 33(1): 168-176 (In Russian with English abstract).

Aslanov, O., Alizade, G. (2018). Predatory Actinedid mites (Acari: Acariformes: Actinedida) of tea plantation of Azerbaijan. Transactions of Pedogogical University, 66(3): 92-97 (In Azeri with English abstract)

Aslanov, O., Alizade, G. (2018). Eritereid Mites (Acari: Acariformes: Actinedida: Erythraeidae). Proceeding of the Azerbaijan Institute of Zoology, 36(1): 129-134 (In Russian with English abstract).

Beron, P. (2008). Acarorum Catalogus I Acariformes: Calyptostomatoidea (Calyptostomatidae), Erythraeoidea (Smarididae, Erythraeidae). Pensoft and Nat. Mus. Natur. Hirst. Sofia: 271 p.

Haitlinger, R. (1987). The genus Leptus Latreille, 1796 and Charletonia Oudemans, 1910 (Acari, Prostigmata, Erythraeidae) in Poland (larvae). Polish Journal of Entomology, 57: 339-349

Jamshidian, M., Noei, J., Reza, A. (2014). First record of the genus Curteria (Acari: Erythraeidae) from Iran. Persian Journal of Acarology, 3(1): 103-106

Makol, J., Sevsay, S. (2015). Abalakeus Southcott, 1994 is a junior synonym of "plume-footed" Eatoniana Cambridge, 1898 (Trombidiformes, Erythraeidae)-evidence from experimental rearing. Zootaxa, 3918(1): 92-112

Noei, J., Ersin, F., Çakmak, İ. (2019). A new larval species of Balaustium (Acari: Actinotrichida: Erythraeidae) from Turkey. Turkish journal of Zoology, 43: 30-42

Wohltmann, A. (2013). Fauna Europaea: Arachnida, Erythraeidae. Fauna Europaea version 2017.06 https://fauna-eu.org

\section{Citation:}

Alizade, G.A. (2020). Erythraeid mites (Acariformes: Actinedida: Erythraeidae) of the Greater Caucasus region of Azerbaijan. Ukrainian Journal of Ecology, 10(1), 199-209. 